\title{
Dihydromyricetin induces apoptosis in a human choriocarcinoma cell line
}

\author{
YANZHEN ZUO ${ }^{1}$, QIAN XU $^{2}$, YANJIE LU $^{3}$, DAYONG SUN ${ }^{4}, \mathrm{KANG} \mathrm{WANG}^{3}$, \\ YUNTAO LEI ${ }^{3}$, XIUJUN LIANG ${ }^{2}$ and YUHONG LI ${ }^{3}$ \\ ${ }^{1}$ Department of Pharmacology, ${ }^{2}$ Research Laboratory and ${ }^{3}$ Department of Pathology, Chengde Medical University; \\ ${ }^{4}$ Department of Tumor Radiation and Chemotherapy Center, The Chengde Central Hospital, \\ Chengde, Hebei 067000, P.R. China
}

Received November 29, 2017; Accepted May 23, 2018

DOI: $10.3892 / \mathrm{ol} .2018 .9220$

\begin{abstract}
Choriocarcinoma is a malignant trophoblastic tumor. The development of novel drugs is required to reduce the toxicity of current multi-agent chemotherapy and to successfully treat chemoresistant cases of the disease. The purpose of the present study was to investigate the effect of dihydromyricetin (DMY) on the human choriocarcinoma cell line, JAr, to identify a novel drug for the treatment of choriocarcinoma. An MTT assay was performed to determine the effects of DMY at different concentrations and for different exposure durations. Flow cytometry and TUNEL assays were performed to detect apoptosis, and western blotting was utilized to investigate the underlying mechanism. The results revealed that DMY significantly inhibited JAr cell viability in a time- and dose-dependent manner. The flow cytometry and TUNEL assays demonstrated that DMY inhibited proliferation by inducing apoptosis. Further analysis by western blotting indicated that the protein expression level of BCL-2 associated X, associated protein increased, while the protein expression levels of BCL-2 and pro-caspase- 3 decreased. These findings suggest that DMY induced apoptosis in human choriocarcinoma JAr cells, through a mitochondrially mediated apoptotic pathway.
\end{abstract}

\section{Introduction}

Choriocarcinoma is a highly malignant trophoblastic pregnancy-associated tumor that often occurs with complete hydatidiform mole $(1,2)$. It grows rapidly and is able to metastasize widely to other organs or tissues through the venous and lymphatic system. Although the complete recovery rate has improved owing to advances in chemotherapy, the toxicity

Correspondence to: Professor Yuhong Li, Department of Pathology, Chengde Medical University, 28 Anyuan Street, Chengde, Hebei 067000, P.R. China

E-mail: youngcheer2003@foxmail.com

Key words: choriocarcinoma, apoptosis, dihydromyricetin, JAr cells, mitochondria and side effects of the drugs used are poorly tolerated (3). Furthermore, $7 \%$ of low-risk patients and $27 \%$ of high-risk patients may exhibit an incomplete response to first-line, single-agent or multi-agent chemotherapy, and may relapse from remission (4). Thus, it remains necessary to develop novel, specific and low-toxicity drugs for the treatment of choriocarcinoma.

Dihydromyricetin (DMY; $\mathrm{C}_{15} \mathrm{H}_{12} \mathrm{O}_{8}$ ), a natural flavonoid, is an active component of extracts from Ampelopsis grossedentata (5). Numerous pharmacological functions of DMY have been reported, including antioxidant, antibacterial, anti-inflammatory, antihypertensive, hepatoprotective and anticancer effects (6-10). The potent in vitro antitumor activity of DMY has also been revealed through the induction of apoptosis in various cell lines, including HepG2 cells, head and neck squamous cell carcinoma, human non-small cell lung cancer and gastric cancer cells (11-14). DMY has been indicated to have antitumor effects in nude mice inoculated with GLC-82 lung cancer cells, as well as nude mice inoculated with Bel-7402 hepatocellular carcinoma cells $(15,16)$. Furthermore, DMY could suppress distant pulmonary metastasis of 4T1 mouse breast carcinoma (17). DMY has been demonstrated to exert a strong antitumor effect with low toxicity $(18,19)$ with a maximum tolerated dose of $5.0 \mathrm{~g} / \mathrm{kg}$ in Wistar mice (20). DMY has been indicated to exhibit antitumor activity in vitro and in vivo without evident toxicity. However, the effects of DMY on human choriocarcinoma remain to be described. In the present study it was revealed that DMY inhibited JAr cell viability in vitro, which indicated that DMY may be a novel drug for the treatment of choriocarcinoma. Subsequently, the antitumor activity of DMY in human choriocarcinoma was determined.

\section{Materials and methods}

Reagents. DMY (>99\% purity) was purchased from the Beijing Hengyuan Qitian Research Institute of Chemical Technology (Beijing, China) and dissolved in DMSO $(<0.05 \%$, v/v, without detectable effects) for all study experiments.

Cell culture. Human fetally derived trophoblast choriocarcinoma JAr cells were obtained from the State Key Laboratory of Reproductive Biology, Institute of Zoology, Chinese Academy 
of Sciences (Shanghai, China). JAr cells were cultured at $37^{\circ} \mathrm{C}$ in $5 \% \mathrm{CO}_{2}$ in DMEM medium (Gibco; Thermo Fisher Scientific, Inc., Waltham, MA, USA) supplemented with 10\% fetal bovine serum (Gibco; Thermo Fisher Scientific, Inc.). The cells were then passaged using $0.25 \%$ trypsin and $0.02 \%$ EDTA (Gibco; Thermo Fisher Scientific, Inc.) when the confluency reached $70-80 \%$.

MTT assay. JAr cells were seeded in 96-well plates and allowed to adhere overnight. When the confluency reached $30-40 \%$, the cells were incubated for 24 or $48 \mathrm{~h}$ with $0,20,40,80$ or $100 \mathrm{mg} / \mathrm{l}$ of DMY in $200 \mu \mathrm{l}$. Each treatment was performed in 6 wells. The cell viability was determined using MTT reagent (Gibco; Thermo Fisher Scientific, Inc.), according the manufacturer's protocol, and the absorbance was determined at a wavelength of $492 \mathrm{~nm}$ by using a Multiskan MK3 microplate reader (Thermo Fisher Scientific Inc., Waltham, MA, USA). The experiment was repeated three times.

Flow cytometry assay. JAr cells were incubated for $48 \mathrm{~h}$ with DMY at the designated concentrations $(0,40,60$ and $100 \mathrm{mg} / \mathrm{l}$ ) and then processed with an AV-FITC kit (BioBox, Ba11100, Nanjing, China), in accordance with the manufacturer's protocol. The samples were analyzed by a FACSCalibur flow cytometer (BD Biosciences, Franklin Lakes, NJ, USA) at $488 \mathrm{~nm}$, in order to quantify the apoptotic rate.

TUNEL assay. Apoptosis of JAr cells was determined using a TUNEL detection kit (Roche Applied Science, Penzberg, Germany) in accordance with the manufacturer's protocol. The JAr cells were incubated for $48 \mathrm{~h}$ with DMY at the designated concentrations $(0,40,60$ and $100 \mathrm{mg} / \mathrm{l})$, fixed with $4 \%$ paraformaldehyde in PBS for $1 \mathrm{~h}$ at room temperature and permeabilized with $0.1 \%$ Triton $\mathrm{X}-100$ in $0.1 \%$ sodium citrate for $2 \mathrm{~min}$ on ice. According to the manufacturer's protocol, a positive control was permeabilized with DNase I recombinant for $10 \mathrm{~min}$ at $15-25^{\circ} \mathrm{C}$ to induce DNA strand breaks. Then the cells were washed in PBS, incubated with the TUNEL reaction mixture for $1 \mathrm{~h}$ at $37^{\circ} \mathrm{C}$ (a negative control incubated with label solution instead of TUNEL reaction mixture), washed again with PBS, and incubated with $0.1 \mu \mathrm{g} / \mathrm{ml}$ DAPI in PBS at $30^{\circ} \mathrm{C}$ for $15-30 \mathrm{~min}$. The samples were analyzed, following a final wash with PBS, under a fluorescence microscope (IX73; Olympus, Tokyo, Japan). The apoptotic rate was quantified by counting the apoptotic cells in six random fields.

Western blot analysis. The JAr cells were incubated for $48 \mathrm{~h}$ with DMY at the designated concentrations $(0,40,60$ and $100 \mathrm{mg} / \mathrm{l})$, collected, and lysed on ice with radioimmunoprecipitation assay buffer (Thermo Fisher Scientific, Inc.). The cell lysates were centrifuged at $14,000 \mathrm{x} \mathrm{g}$ for $10 \mathrm{~min}$ at $4^{\circ} \mathrm{C}$. The protein concentration was quantified by the bicinchoninic acid assay (BCA; Pierce; Thermo Fisher Scientific, Inc.). Equal amounts $(30 \mu \mathrm{g})$ of protein were separated by $12 \%$ SDS-PAGE and then transferred onto a polyvinylidene difluoride (PVDF) membrane. The membranes were blocked with 5\% non-fat milk and incubated with the following primary antibodies (diluted 1:1,000) overnight at $4^{\circ} \mathrm{C}$ : $\beta$-actin (mouse anti-human monoclonal; cat. no. sc-130065; Santa Cruz Biotechnology, Inc., Dallas, TX, USA),

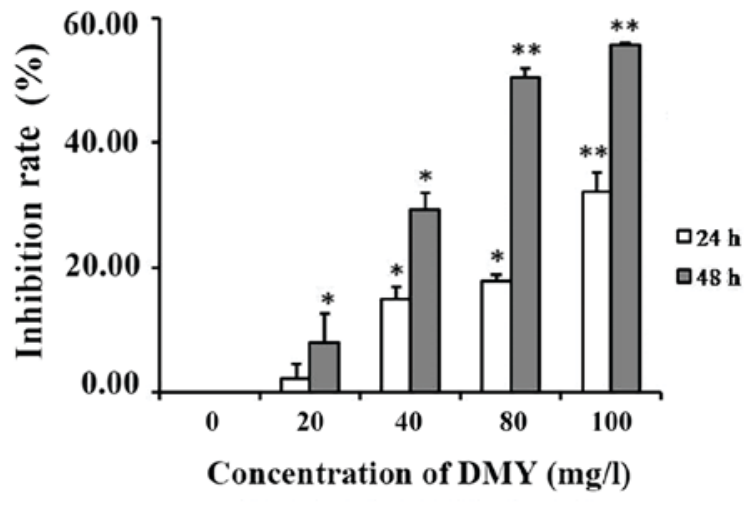

Figure 1. The effects of DMY on the viability of JAr cells. The cell viability was measured by MTT assay and the experiment was repeated three times. The cells were incubated with 0, 40, 80 or $100 \mathrm{mg} / 1 \mathrm{DMY}$ for 24 and $48 \mathrm{~h}$. The viability of JAr cells was time- and dose-dependently reduced. ${ }^{*} \mathrm{P}<0.05$ and ${ }^{* *} \mathrm{P}<0.01$, vs. 0 mg/l DMY group. DMY, dihydromyricetin.

Bax (mouse anti-human monoclonal; cat. no. ab77566; Abcam, Cambridge, UK), Bcl-2 (rabbit anti-human polyclonal; cat. no. E1A6139; Enogene, Nanjing, China), poly (ADP-ribose) polymerase (Parp), pro-caspase-3 and cleaved caspase-3 (rabbit anti-human polyclonal; cat. nos. 9661s and 14220s, respectively; Cell Signaling Technology, Inc., Danvers, MA, USA). The membranes were then washed in TBS-Tween $(0.05 \%)$ buffer and incubated with a secondary antibody (peroxidase-conjugated Affinipure goat anti-mouse IgG, cat. no. ab6728; peroxidase-conjugated Affinipure goat anti-rabbit IgG, cat. no. ab6721; dilution 1:5,000; Abcam) for $1 \mathrm{~h}$ at room temperature. Immunoreactive bands were detected by enhanced chemiluminescence (Pierce; Thermo Fisher Scientific, Inc.) and imaged using the Tanon-6100 Chemiluminescent Imaging system (Tanon Science and Technology Co., Ltd., Shanghai, China). The band densities were calculated with Quantity One software 4.6.2 (Bio-Rad Laboratories, Inc., Hercules, CA, USA).

Statistical analysis. Statistical analysis was performed using SPSS 19.0 (IBM Corp., Armonk, NY, USA). All data are expressed as the mean \pm standard deviation. One-way analysis of variance was used to make comparisons between groups. The pairwise comparison of means among groups was performed using the Student-Newman-Keuls method. $\mathrm{P}<0.05$ was considered to indicate a statistically significant difference.

\section{Results}

DMY inhibits the viability of JAr cells. An MTT proliferation assay was performed to evaluate the influence of $0,20,40,80$ and $100 \mathrm{mg} / \mathrm{l} \mathrm{DMY}$ on the cellular viability of JAr cells at 24 and $48 \mathrm{~h}$ (Fig. 1). As indicated, the viability of JAr cells was time- and dose-dependently reduced following treatment with DMY in comparison with the control cells $(\mathrm{P}<0.05$; Fig. 1$)$

DMY induced JAr cell apoptosis. The present study investigated apoptosis following incubation with different concentrations of DMY for $48 \mathrm{~h}$. The quantitative analysis of apoptosis by flow cytometry using Annexin V/PI dual staining revealed that the apoptotic rate increased in a dose-dependent manner (Fig. 2A). 
A
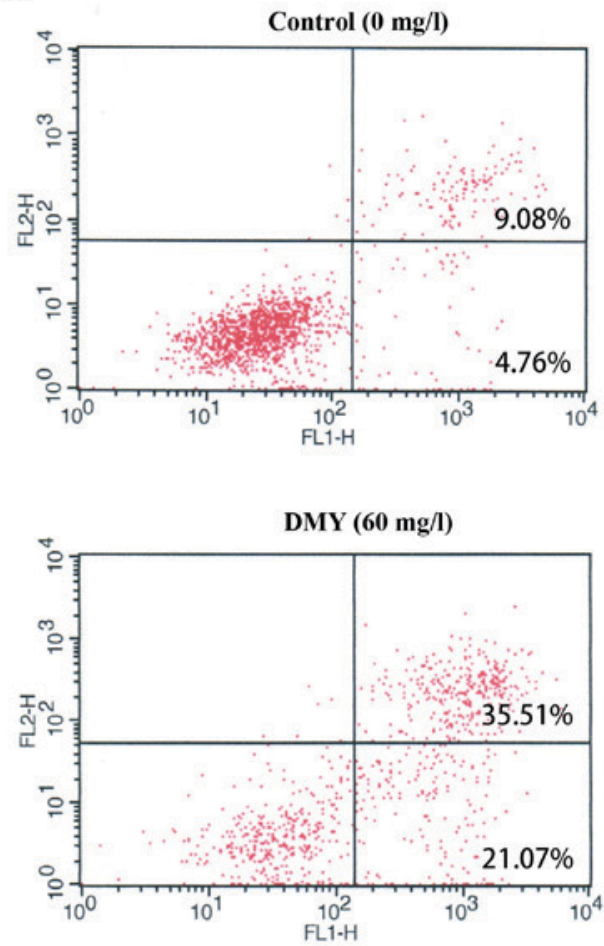

B

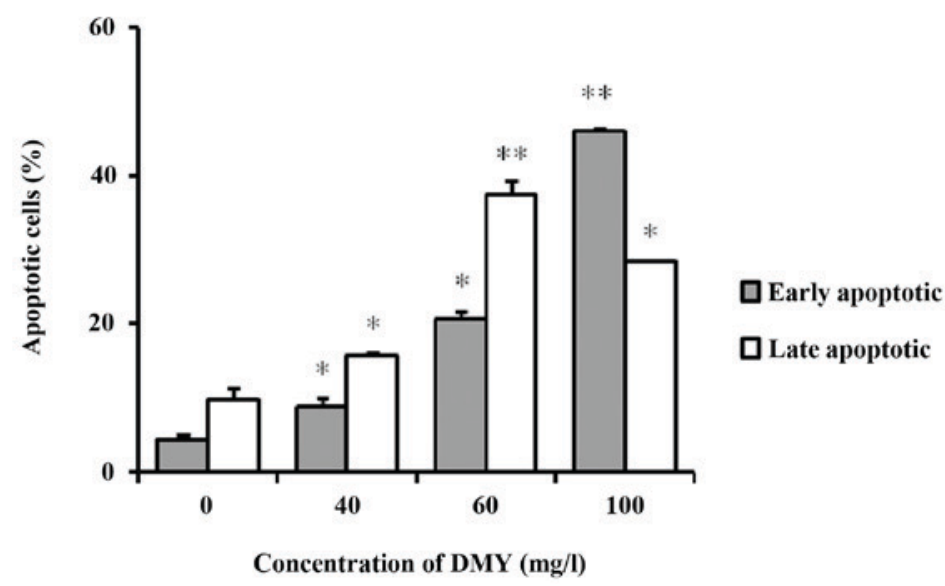

Figure 2. The cells were incubated with 0, 40, 60 or 100 mg/l DMY for 48 h. (A) Apoptosis was detected using an FITC Annexin V-PI Apoptosis detection kit and measured using flow cytometry (B). The data revealed that DMY induced JAr cell apoptosis in a dose-dependent manner. ${ }^{*} \mathrm{P}<0.05$ and ${ }^{* *} \mathrm{P}<0.01 \mathrm{vs} .0 \mathrm{mg} / 1$ DMY group. DMY, dihydromyricetin.

At $0,40,60$ and $100 \mathrm{mg} / \mathrm{l}$ of DMY the proportion of apoptotic cells was $14.2 \pm 1.69,24.43 \pm 1.72,58 \pm 2.08$ and $74.42 \pm 0.41 \%$, respectively $(\mathrm{P}<0.05$; Fig. $2 \mathrm{~B})$.

The DMY-induced apoptosis of JAr cells was also confirmed by TUNEL staining. In the control group, few apoptotic JAr cells were observed, but DMY treatment resulted in a dose-dependent increase in TUNEL-positive JAr cells $(\mathrm{P}<0.05$; Fig. 3$)$.

$D M Y$ induced apoptosis increases the Bax/Bcl-2 ratio and decreases pro-caspase-3 expression. With DMY treatment, the protein expression level of Bax increased, whereas the expression level of Bcl-2 decreased in a dose-dependent manner (Fig. 4A). The Bax/Bcl-2 ratio significantly increased with DMY treatment in comparison with that of the control group $(\mathrm{P}<0.05)$, particularly in the $100 \mathrm{mg} / 1$ group $(\mathrm{P}<0.01$; Fig. 4B).
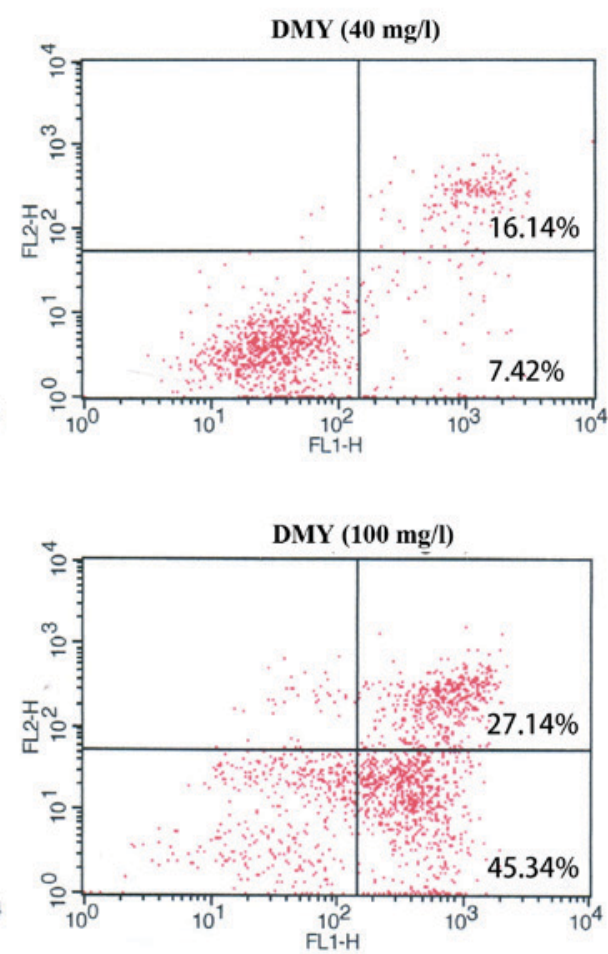


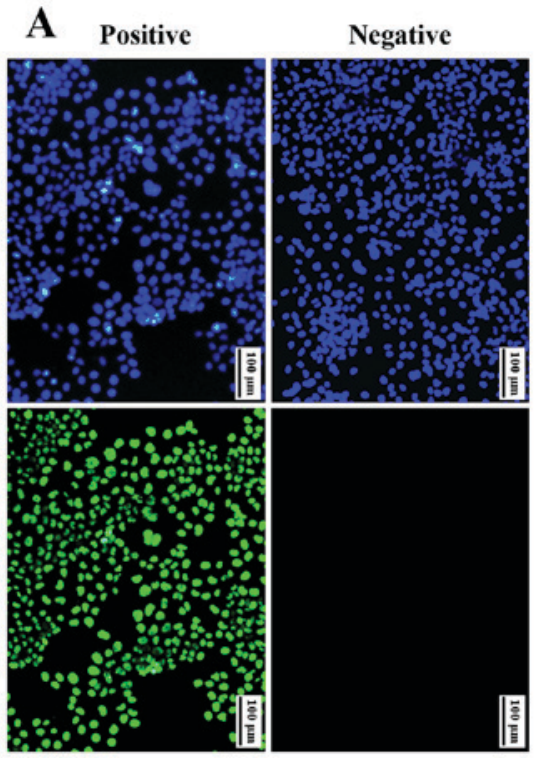

C
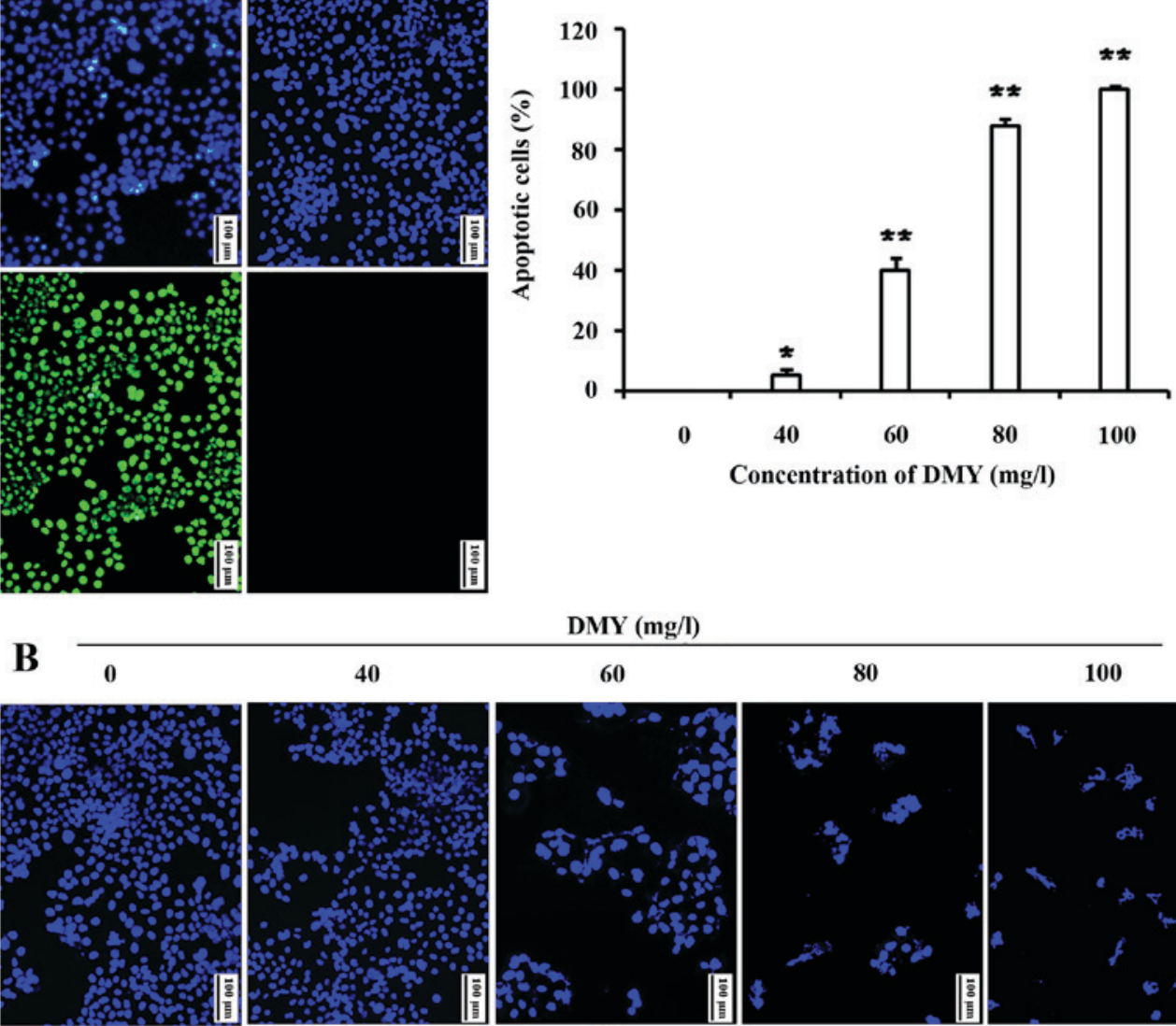

$\operatorname{DMY}(\mathrm{mg} / \mathbf{l})$

60

80

100
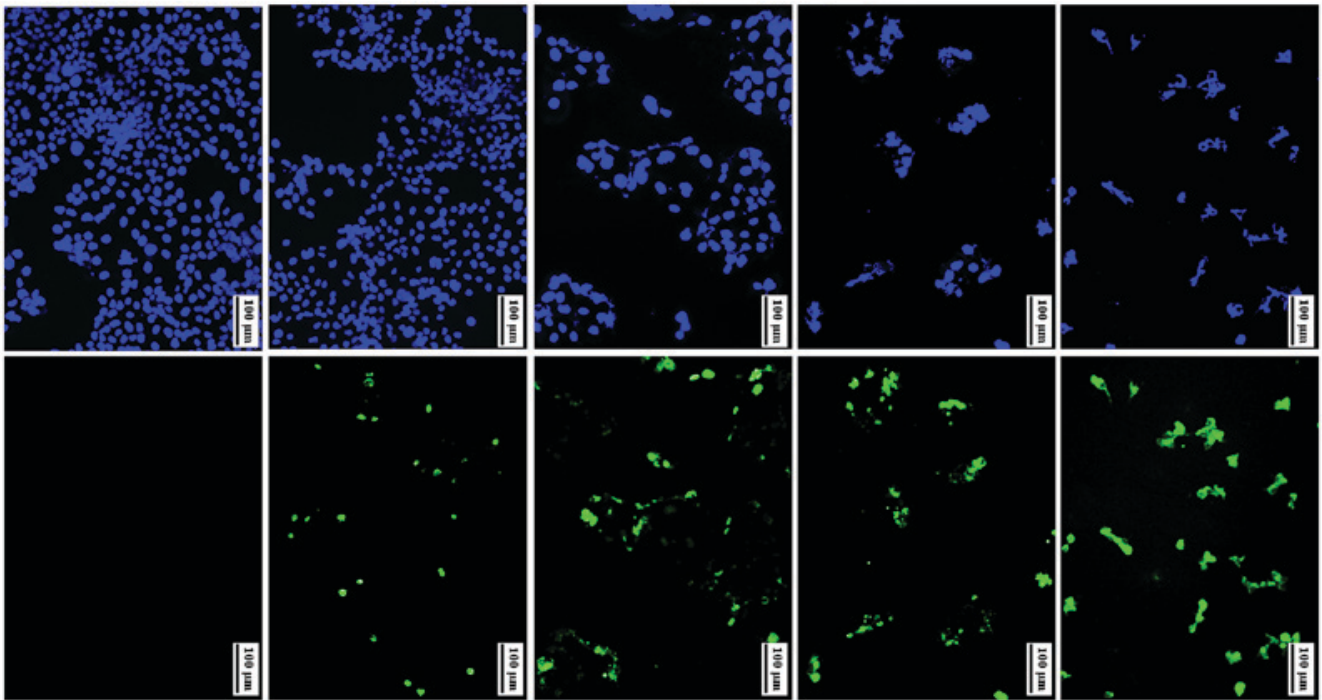

Figure 3. The effects of DMY on JAr cells apoptosis. The exposure time to TUNEL was the same for all groups. Apoptosis was determined by TUNEL staining (green staining) and cell total number was determined by DAPI staining (blue staining). (A) Positive and negative groups. In accordance with the Tunel manufacturer's protocol, for the negative control Label solution was added instead of TUNEL reaction mixture, so the negative control group exhibits no green staining, with the aim of eliminating false-positives. A positive control was permeabilized with DNase I recombinant for 10 min at $15-25^{\circ} \mathrm{C}$ to induce DNA strand breaks. The positive control group thus exhibits all green staining, with the aim of eliminating false-negatives. (B) Changes in the nuclear chromatin were observed in JAR cells using TUNEL/DAPI staining. (C) Quantification of TUNEL-positive cells following treatment with 0, 40, 60, 80 or $100 \mathrm{mg} / \mathrm{l} \mathrm{DMY}$. TUNEL staining indicated that the proportion of apoptotic JAr cells increased in a dose-dependent manner with DMY treatment. ${ }^{*} \mathrm{P}<0.05$ and ${ }^{* *} \mathrm{P}<0.01$ vs. $0 \mathrm{mg} / \mathrm{l}$ group. Scale bar, $100 \mu \mathrm{m}$. DMY, dihydromyricetin.

apoptosis and were performed at $48 \mathrm{~h}$ of DMY treatment, the time point at which the inhibitory effect was most significant. The results revealed that DMY induced the apoptosis of JAr cells in a dose-dependent manner, suggesting that DMY reduced proliferation through the induction of apoptosis.

Apoptosis is a form of programmed cell death that serves an important role in the development and treatment of tumors (21). The regulatory pathways of apoptosis, which include a number of gene families, orchestrate the specific morphological and biochemical changes that occur during the apoptotic process (22). In the mitochondrial pathway to cell death, the apoptotic threshold is set by interactions on the mitochondrial outer membrane between three functionally and structurally distinct subgroups of the Bcl-2 protein family: Bcl-2 homology 3 (BH3)-only proteins, which convey signals to initiate apoptosis, pro-survival cell guardians, including Bcl-2 itself, and pro-apoptotic effector proteins, including Bax and BCl-2 antagonist/killer (23). Bax forms oligomers, which permeabilize the mitochondrial outer membrane, releasing cytochrome $\mathrm{c}$ into the cytosol and activating the effector caspases (24-26). Bcl-2 binds to Bax and inhibits its pro-apoptotic activity (27). To investigate the mechanism by which DMY triggers apoptosis, western blot analysis was performed to analyze the potential proteins involved. The results revealed that DMY treatment increased the protein expression level of Bax and decreased that of Bcl-2. Although 
A

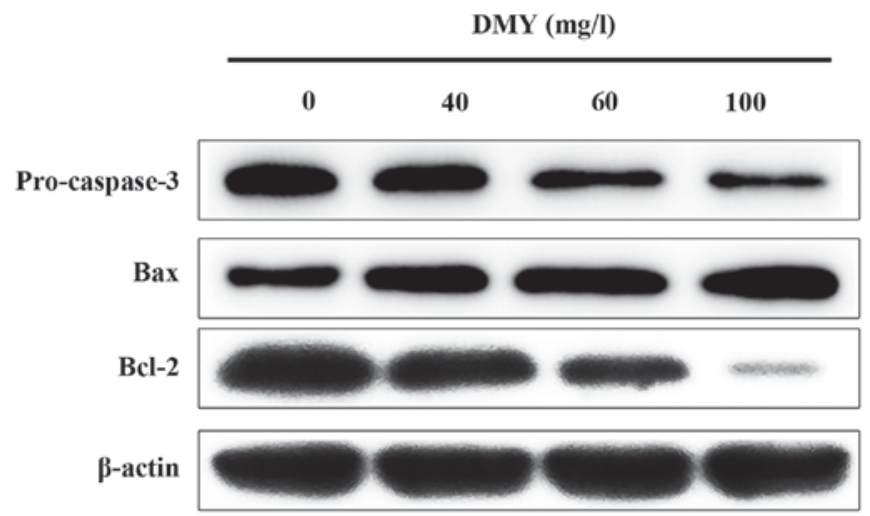

B
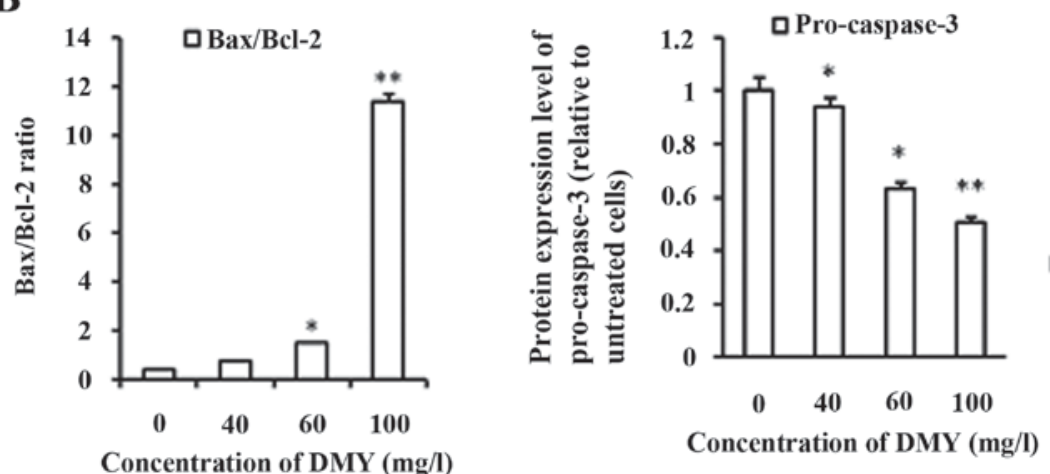

Figure 4. Western blot analysis of the effects of DMY on protein expression levels in JAr cells. (A) Western blot analysis of cells treated 0 , 40,60 or 100 mg/1 DMY for 48 h. (B) Bar charts indicating DMY increased the expression level of Bax and decreased that of Bcl-2 (arbitrary units). The protein expression level of pro-caspase- 3 decreased, which was indicative of its cleavage and activation. The experiment was performed in triplicate. ${ }^{*} \mathrm{P}<0.05$ and ${ }^{* *} \mathrm{P}<0.01 \mathrm{vs} .0 \mathrm{mg} / 1$ group. DMY, dihydromyricetin; Bax, Bcl-2 associated X.

cleaved caspase-3 was not detected, the expression level of pro-caspase-3 decreased, which indicated that it was cleaved and activated. Pro-caspase- 3 is processed by autoproteolytic cleavage (or by one or more other proteases in transit) that leads to the assembly of the active heterotetrameric enzyme (28). The activation of caspase- 3 raises the question of whether this protease is required for cell death (28).

It was concluded that DMY induced apoptosis in the JAr cell line and it was confirmed that the apoptosis was mitochondrially mediated by changes in the expression level of the $\mathrm{Bax} / \mathrm{Bcl}-2$ protein ratio and decreased protein expression of pro-caspase-3. To determine whether DMY could be a novel therapeutic drug for choriocarcinoma, further studies are being performed, in which the promising prospects of the present study will be reported in detail.

\section{Acknowledgments}

Not applicable.

\section{Funding}

The present study was supported by the Key Subjects in Universities and Colleges of Hebei Province of China [Pathology and Pathophysiology; grant no. JiJiaoGao(2013)4], the Excellent Innovation Talent Support Plan of Hebei Education Department (grant no. SLRC2017018) and the Project in Hebei province Department of Education (grant no. QN2016012).

\section{Availability of data and materials}

The datasets used and/or analyzed during the present study are available from the corresponding author on reasonable request.

\section{Authors' contributions}

YZZ designed the study, performed the experiment, analyzed and interpreted the data and prepared the first draft of the manuscript. QX, YJL and DYS contributed to the design, data analysis and revision of the manuscript. KW and YTL performed the experiment. XJL contributed to flow cytometry assay. YHL developed the concept of the study, performed the data analysis and revised the manuscript. All authors read and approved the final manuscript.

\section{Ethics approval and consent to participate}

Not applicable.

\section{Patient consent for publication}

Not applicable.

\section{Competing interests}

The authors declare that they have no competing interests. 


\section{References}

1. Xu Q, Tan Y, Zhang K and Li Y: Crosstalk between p38 and Smad3 through TGF- $\beta 1$ in JEG-3 choriocarcinoma cells. Int J Oncol 43: 1187-1193, 2013.

2. Tan Y, Xu Q, Li Y, Mao X and Zhang K: Crosstalk between the p38 and TGF- $\beta$ signaling pathways through T $\beta R I, T \beta R I I$ and Smad3 expression in plancental choriocarcinoma JEG-3 cells. Oncol Lett 8: 1307-1311, 2014.

3. Yamamoto E, Ino K, Yamamoto T, Sumigama S, Nawa A, Nomura S and Kikkawa F: A pure nongestational choriocarcinoma of the ovary diagnosed with short tandem repeat analysis: Case report and review of the literature. Int J Gynecol Cancer 17: 254-258, 2007.

4. Kobayashi Y, Shimizu T, Naoe H, Ueki A, Ishizawa J, Chiyoda T, Onishi N, Sugihara E, Nagano O, Banno K, et al: Establishment of a choriocarcinoma model from immortalized norma extravillous trophoblast cells transduced with HRASV12. Am J Pathol 179: 1471-1482, 2011.

5. Wu S, Liu B, Zhang Q, Liu J, Zhou W, Wang C, Li M, Bao S and Zhu R: Dihydromyricetin reduced Bcl-2 expression via p53 in human hepatoma HepG2 cells. PLoS One 8: e76886, 2013.

6. Ye J, Guan Y, Zeng S and Liu D: Ampelopsin prevents apoptosis induced by $\mathrm{H} 2 \mathrm{O} 2$ in MT-4 lymphocytes. Planta Med 74: 252-257, 2008.

7. Wu Y, Bai J, Zhong K, Huang Y and Gao H: A dual antibacterial mechanism involved in membrane disruption and DNA binding of 2R,3R-dihydromyricetin from pine needles of Cedrus deodara against Staphylococcus aureus. Food Chem 218 463-470, 2017

8. Xu B, Huang S, Wang C, Zhang H, Fang S and Zhang Y: Anti-inflammatory effects of dihydromyricetin in a mouse model of asthma. Mol Med Rep 15: 3674-3680, 2017.

9. Liu B, Zhou W, Chen X, Xu F, Chen Y, Liu J, Zhang Q, Bao S, Chen N, Li M and Zhu R: Dihydromyricetin induces mouse hepatoma Hepal- 6 cell apoptosis via the transforming growth factor- $\beta$ pathway. Mol Med Rep 11: 1609-1614, 2015.

10. Wang JT, Jiao P, Zhou Y and Liu Q: Protective effect of dihydromyricetin against lipopolysaccharide-induced acute kidney injury in a rat model. Med Sci Monit 22: 454-459, 2016.

11. Fan TF, Wu TF, Bu LL, Ma SR, Li YC, Mao L, Sun ZJ and Zhang WF: Dihydromyricetin promotes autophagy and apoptosis through ROS-STAT3 signaling in head and neck squamous cell carcinoma. Oncotarget 7: 59691-59703, 2016.

12. Zhang Z, Zhang H, Chen S, Xu Y, Yao A, Liao Q, Han L, Zou Z and Zhang X: Dihydromyricetin induces mitochondria-mediated apoptosis in HepG2 cells through down-regulation of the Akt/Bad pathway. Nutr Res 38: 27-33, 2017.

13. Ji FJ, Tian XF, Liu XW, Fu LB, Wu YY, Fang XD and Jin HY: Dihydromyricetin induces cell apoptosis via a p53-related pathway in AGS human gastric cancer cells. Genet Mol Res 14: 15564-15571, 2015.

14. Kao SJ, Lee WJ, Chang JH, Chow JM, Chung CL, Hung WY and Chien MH: Suppression of reactive oxygen species-mediated ERK and JNK activation sensitizes dihydromyricetin-induced mitochondrial apoptosis in human non-small cell lung cancer. Environ Toxicol 32: 1426-1438, 2017.
15. Zeng S, Liu D, Ye Y, Wang L and Wang W: Anti-tumor effects of ampelopsin on human lung cancer GLC-82 implanted in nude mice. Zhong Yao Cai 27: 842-845, 2004 (In Chinese).

16. Luo GQ, Zeng S and Liu DY: Inhibitory effects of ampelopsin on angiogenesis. Zhong Yao Cai 29: 146-150, 2006 (In Chinese).

17. Zhou FZ and Zhang XF: Suppression of distant pulmonary metastasis of 4T1 mice breast carcinoma by dihydromyricetin administration. Chin J Clinicians(Electronic Ed) 8: 1674-1678, 2014 (In Chinese)

18. Jiang L, Zhang Q, Ren H, Ma S, Lu C, Liu B, Liu J, Liang J, Li M and Zhu R: Dihydromyricetin enhances the chemo-sensitivity of nedaplatin via regulation of the $\mathrm{p} 53 / \mathrm{Bcl}-2$ pathway in hepatocellular carcinoma cells. PLoS One 10: e0124994, 2015.

19. Wong IL, Wang BC, Yuan J, Duan LX, Liu Z, Liu T, Li XM, Hu X, Zhang XY, Jiang T, et al: Potent and nontoxic chemosensitizer of P-Glycoprotein-mediated multidrug resistance in cancer: Synthesis and evaluation of methylated epigallocatechin, gallocatechin, and dihydromyricetin derivatives. J Med Chem 58: 4529-4549, 2015.

20. Su DL, Huang JH and Yao MJ: The acute toxicological evaluation of dihydromyricetin and its control effect for alcoholic hepatic injury. Hun Agricultural Sci: 90-93, 2009 (In Chinese).

21. Biaglow JE and Miller RA: The thioredoxin reductase/thioredoxin system: Novel redox targets for cancer therapy. Cancer Biol Ther 4: 6-13, 2005.

22. Kiraz Y, Adan A, Kartal Yandim M and Baran Y: Major apoptotic mechanisms and genes involved in apoptosis. Tumour Biol 37: 8471-8486, 2016.

23. Czabotar PE, Lessene G, Strasser A and Adams JM: Control of apoptosis by the BCL-2 protein family: Implications for physiology and therapy. Nat Rev Mol Cell Biol 15: 49-63, 2014.

24. Ghoneum M, Matsuura M, Braga M and Gollapudi S: S. cerevisiae induces apoptosis in human metastatic breast cancer cells by altering intracellular $\mathrm{Ca} 2+$ and the ratio of $\mathrm{Bax}$ and $\mathrm{Bcl}-2$. Int J Oncol 33: 533-539, 2008.

25. Kluck RM, Bossy-Wetzel E, Green DR and Newmeyer DD: The release of cytochrome c from mitochondria: A primary site for Bcl-2 regulation of apoptosis. Science 275: 1132-1136, 1997.

26. Bar-Am O, Weinreb O, Amit T and Youdim MB: Regulation of $\mathrm{Bcl}-2$ family proteins, neurotrophic factors, and APP processing in the neurorescue activity of propargylamine. FASEB J 19: 1899-1901, 2005.

27. Prenek L, Boldizsár F, Kugyelka R, Ugor E, Berta G, Németh P and Berki T: The regulation of the mitochondrial apoptotic pathway by glucocorticoid receptor in collaboration with $\mathrm{Bcl}-2$ family proteins in developing T cells. Apoptosis 22: 239-253, 2017.

28. Porter AG and Jänicke RU: Emerging roles of caspase-3 in apoptosis. Cell Death Differ 6: 99-104, 1999.

This work is licensed under a Creative Commons Attribution-NonCommercial-NoDerivatives 4.0 International (CC BY-NC-ND 4.0) License. 